\title{
Interpretation of Subtle Interstitial Chest Abnormalities: Conventional Radiography Versus High-Resolution Storage-Phosphor Radiography-A Preliminary Study
}

\author{
Junpei Ikezoe, Nobuaki Kohno, Shoji Kido, Noriyuki Takeuchi, Takeshi Johkoh, Jun Arisawa, \\ and Takahiro Kozuka
}

To evaluate the reliability of digital chest radiography in diagnosing subtle interstitial lung abnormalities, we performed several clinical studies including a comparison of conventional screen-film radiography and storage-phosphor radiography ( $2 \mathrm{~K} \times 2 \mathrm{~K}$ pixels, 10 bit), and a comparison of conventional screen-film radiography and film-digitized radiography $(2 \mathrm{~K} \times 2 \mathrm{~K}$ pixels, 10 bit). From these previous studies, a spatial resolution of $0.2-\mathrm{mm}$ pixel size was considered inadequate to diagnose subtle interstitial lung diseases. Under these circumstances, the newly developed Fuji Computed Radiography system (FCR 9000; Fuji Photo Film, Tokyo, Japan) has recently become available. This system provides $0.1-\mathrm{mm}$ pixel size $(4 \mathrm{~K} \times 5 \mathrm{~K}$ pixels, 10 -bit depth) and life-size hard copies (14 $\times 17$ inches). To evaluate the reliability of new high-resolution storagephosphor radiography (FCR 9000) in diagnosing simulated subtle interstitial abnormalities (including simulated lines, micronodules, and groundglass opacities), the differences among radiologists in interpreting conventional screen-film radiographs and life-size highresolution storage-phosphor radiographs were studied. Observation was made by eight experienced chest radiologists, and receiver-operating characteristic (ROC) analysis was performed. There was no significant difference in detecting in subtle simulated interstitial abnormalities between conventional film-screen radiography and high-resolution storage-phosphor radiography. For all three types of abnormalities, there was no significant difference between conventional and storage-phosphor radiography. In conclusion, the high-resolution storage-phosphor chest radiography (0.1-mm pixel size, 10-bit depth) may be substituted for conventional chest radiography in the detection of subtle interstitial abnormalities.

Copyright 1995 by W.B. Saunders Company

KEY WORDS: storage-phosphor radiography, highresolution computed radiography, subtle interstitial lung abnormalities.

$\mathbf{D}^{1}$ IGITAL CHEST imaging has various aspects including methods of obtaining digital images, spatial resolution requirement, postprocessing, method of display, and computeraided diagnosis. There are many reports for each topic, but for some topics, there is no worldwide consensus to date. As for the spatial resolution requirement of chest radiography, there is no final consensus. Some authors reported that a $0.4-\mathrm{mm}$ pixel size was sufficient for daily clinical practice, ${ }^{1}$ whereas others reported that a $0.2-\mathrm{mm}$ pixel size was necessary. ${ }^{2}$ Based on a study using film-digitization radiography, MacMahon et $\mathrm{al}^{3}$ reported that the closer the pixel size came to $0.1 \mathrm{~mm}$, the better the diagnostic accuracy was. As for the relationship between localization of the lesion and diagnostic accuracy, the following temporary consensus has been obtained. For lesions located in lowdensity regions such as the mediastinum, digital imaging with high-frequency edge enhancement provided equal or better information than conventional chest radiography. ${ }^{2,4}$ For lesions in the lung parenchyma, especially for large abnormalities such as consolidation, atelectasis, mass and nodule, and apparent interstitial abnormalities, digital imaging provided nearly equal information to conventional chest radiography. However, for subtle interstitial abnormalities, digital imaging was inferior to conventional chest radiography. ${ }^{5}$ At present, clinically acceptable digital systems for chest radiography are storagephosphor radiography and film digitization system. As to the display method, hard copy is superior to a video display terminal, especially for subtle abnormalities and emphysematous change. ${ }^{6,7}$

Under these circumstances, we performed several clinical studies of the special resolution requirement for digital chest radiography using Fuji Computed Radiography (FCR) and filmdigitized radiography. Our studies estimated responses to the following questions: (1) Which pixel size is required for a totally digital radiol-

From the Department of Radiology, Osaka University Medical School, Osaka, Japan.

$A$ portion of this report appeared in Radiology (Kido $S$, Ikezoe J, Takeuchi N, et al, Radiology 187:527-533, 1993 and Kido S, Ikezoe J, Takeuchi N, et al, Radiology 192:171-176, 1994). It is used with permission of RSNA publications.

Address reprint requests to Junpei Ikezoe, MD, Department of Radiology, Osaka University Medical School, 2-2, Yamadaoka, Suita city, Osaka 565, Japan.

Copyright $\odot 1995$ by W.B. Saunders Company

0897-1889/95/0801-1009\$3.00/0 
ogy department? (2) Can conventional radiography can be replaced by FCR? (3) How about film-digitization systems?

\section{CONVENTIONAL SCREEN-FILM RADIOGRAPHY VERSUS FCR}

The first study was "Interpretation of subtle interstitial abnormalities: Conventional vs storage phosphor radiography (FCR $2 \mathrm{~K} \times 2 \mathrm{~K}$ matrix, 10 bits depth)." 8 To evaluate the reliability of FCR $(2 \mathrm{~K} \times 2 \mathrm{~K}$ pixels) for diagnosing subtle interstitial abnormalities, we performed a comparative study with conventional screen-film radiography, life-size $(14 \times 14$ inches $) \mathrm{FCR}$, and minified FCR $2 / 3(9 \times 9$ inches $)$. FCR system we used was FCR 7000 (Fuji Photo Film, Tokyo, Japan) which provided a $2 \mathrm{~K}-\times 2 \mathrm{~K}$-pixel matrix and 10-bit depth, so pixel size was 0.2 $\mathrm{mm}$. Forty patients with subtle interstitial lesions including fine reticular lesions, ill-defined faint small nodules, and groundglass attenuation and 40 patients with normal chests were evaluated by 14 observers ( 7 experienced chest radiologists and 7 residents). ROC analysis was performed. For all 14 observers, there were no significant differences among the three imaging modalities (Fig 1). However, for the 7 experienced chest radiologists, conventional chest radiography was significantly superior to the two types of FCR (Fig 2). Furthermore, the best

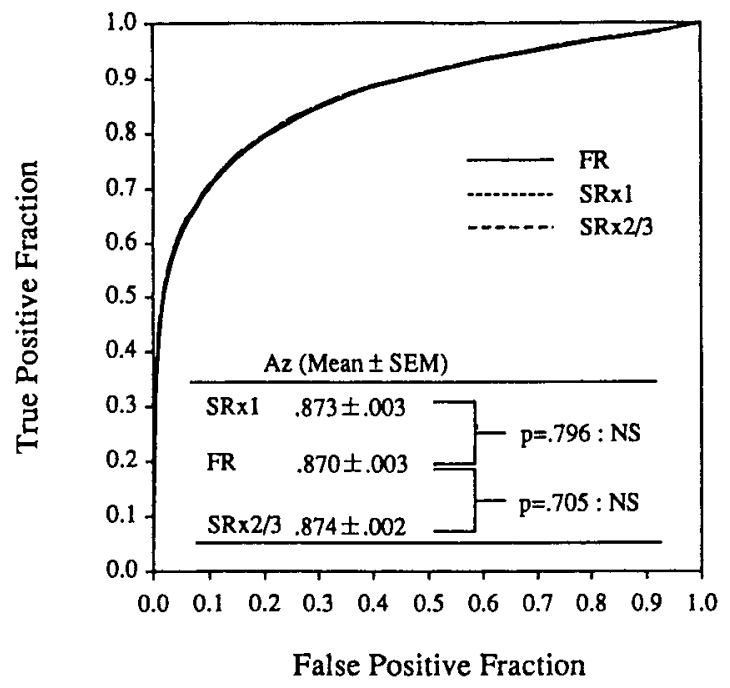

Fig 1. Composite ROC curves of 14 observers. No significant differences in observer performance were observed among conventional film radiographs (FR), full-size storage-phosphor images (SR $\times 1$ ), and minified storage-phosphor images $(S R \times 2 / 3)$. (Reprinted with permission from Kido et al $\left.{ }^{8}\right)$

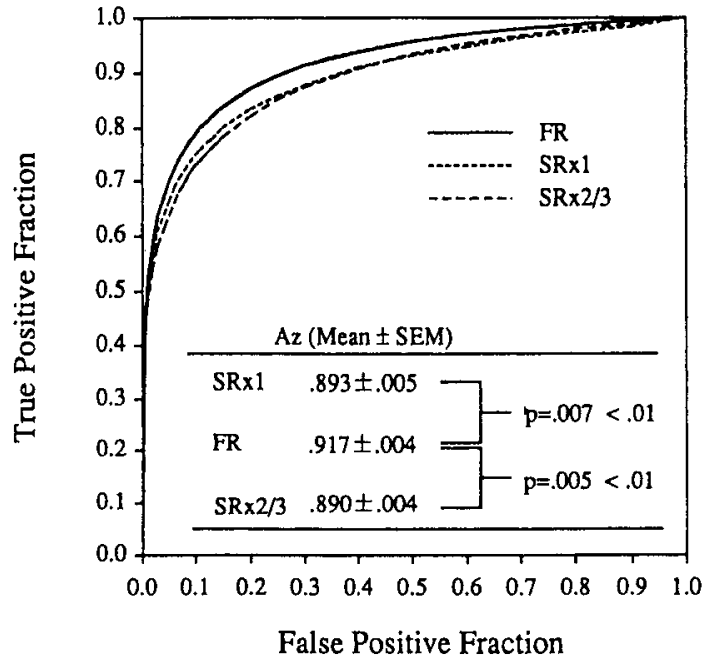

Fig 2. Composite ROC curves of seven chest radiologists. Significant differences in observer performance were observed between conventional radiographs (FR) and two formats of storage-phosphor images (SR $\times 1, S R \times 2 / 3$ ). (Reprinted with permission from Kido et al. ${ }^{8}$ )

perceptual accuracy was obtained with conventional screen-film radiography evaluated by chest radiologists. We concluded that critically important information for chest radiologists may be lost with FCR $(2 \mathrm{~K} \times 2 \mathrm{~K}$ pixels $) .{ }^{8}$

\section{CONVENTIONAL SCREEN-FILM RADIOGRAPHY VERSUS FILM-DIGITIZED RADIOGRAPHY}

The second study we performed was "Interpretation of subtle interstitial abnormalities: Conventional vs film digitized radiography." To evaluate film-digitized radiography for diagnosing subtle interstitial abnormalities, we performed a comparative study with conventional screen-film radiography and film-digitized radiography. The film-digitization system we used was a Laser optical film digitizer that provided a $2 \mathrm{~K}-\times 2 \mathrm{~K}$-pixel matrix and 10-bit depth, so pixel size was $0.175 \mathrm{~mm}$. The same 40 patients with subtle interstitial lesion in the previous study and 40 patients with normal chests, were evaluated by 10 observers. The 10 observers consisted of 5 experienced chest radiologists and 5 residents. ROC analysis was performed. For all observers, no significant differences were found between conventional screen-film radiography and film-digitized radiography (Fig 3). However, for chest radiologists, conventional chest radiography was significantly superior to film-digitized radiography (Fig 4). In conclu- 


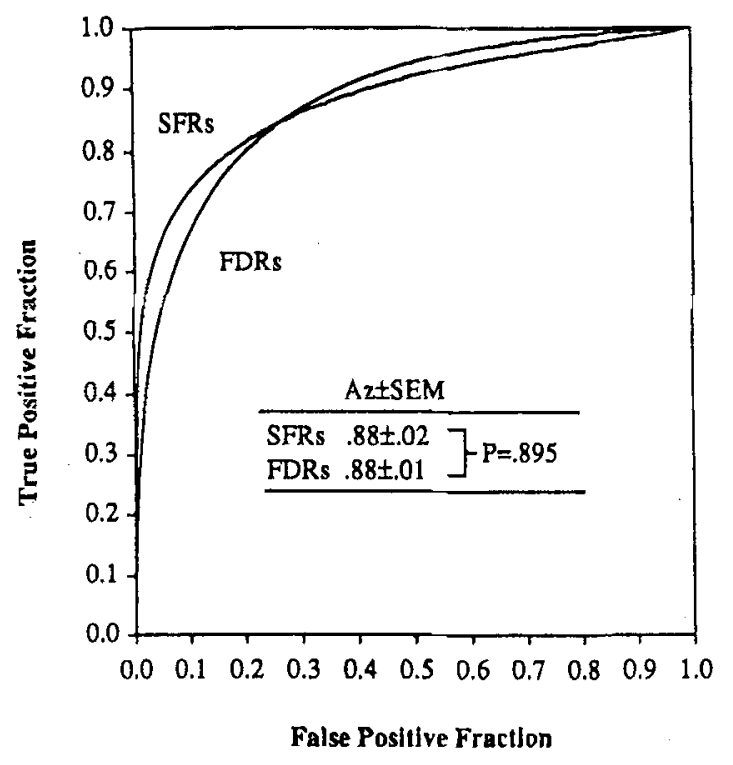

Fig 3. Composite ROC curves of all observers. No statistically significant differences in observer performance were found between conventional screen-film radiographs (SFRs) and film-digitized radiographs (FDRs). (Reprinted with permission from Kido et al. ${ }^{9}$ )

sion, film-digitized radiography with an 0.175 $\mathrm{mm}$ pixel size may be inadequate compared with conventional screen-film radiography for diagnosing subtle interstitial lung abnormalities. ${ }^{9}$

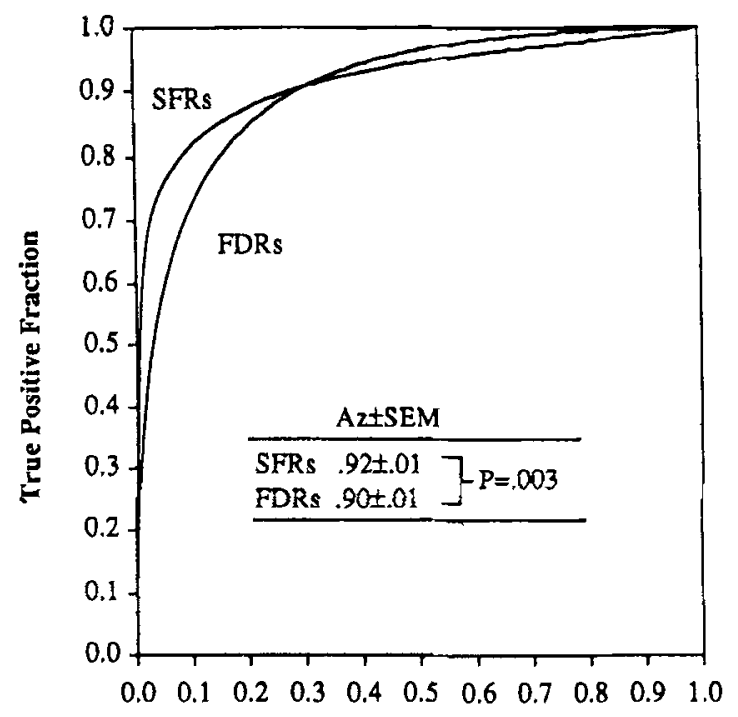

False Positive Fraction

Fig 4. Composite ROC curves of five chest radiologists. Statistically significant differences were observed between conventional screen-film radiographs (SFRs) and film-digitized radiographs (FDRs) $(P=.003)$. (Reprinted with permission from Kido et al. $\left.{ }^{9}\right)$

\section{CONVENTIONAL SCREEN-FILM RADIOGRAPHY VERSUS HIGH-RESOLUTION FCR}

Fuji Photo Film Co investigated a new FCR system that provided a $4 \mathrm{~K}-\times 5 \mathrm{~K}$-pixel matrix, 10-bit depth, and life-size hard copies $(14 \times 14$ inches). Our recent study was "Interpretation of subtle interstitial chest abnormalities: Conventional radiography vs high-resolution storagephosphor radiography." To evaluate the reliability of new high-resolution storage-phosphor radiography in diagnosing simulated subtle interstitial abnormalities (including simulated lines, micronodules, and groundglass opacities), the differences among radiologists in interpreting conventional screen-film radiographs and lifesize high-resolution storage-phosphor radiographs were studied.

The study population consisted of 16 healthy volunteers. Each of them had a conventional radiograph and a storage-phosphor radiograph obtained within minutes of each other. For both, a template containing simulated lesions was placed on an upright Bucky chest unit, and the volunteer was positioned in front of it to superimpose the template onto the chest film in correct registration. The same template in an unaltered position was used for both the conventional and the storage-phosphor radiograph in each volunteer. The template consisted of a polyester film base divided into 15 fields by copper wire, and this was placed onto the chest unit. Three types of simulated abnormalities were used for this study (Fig 5). Fine linear lesions were simulated by thin copper sheets or thin stainless steel wires. Micronodules were simulated by coral sand, and goundglass opacities were made of a mixture of clay and barium sulfate or sponge with olive oil. Three different severity of lesions were used for each type of abnormality. Three types of simulated abnormalities were taped onto each divided field. Six to nine lesions were used with each volunteer. Thus, each field was completely empty or contained one type of lesion.

Both conventional and digital images were obtained under these conditions (Figs 6 and 7). For conventional chest radiographs, Du Pont (Wilmington, DE) Cr-4d films and BF-III screen were used. For storage phosphor radiographs, a new type of FCR (FCR 9000 type) was used, which provided $4 \mathrm{~K}-\times 5 \mathrm{~K}$-pixel matrix and 


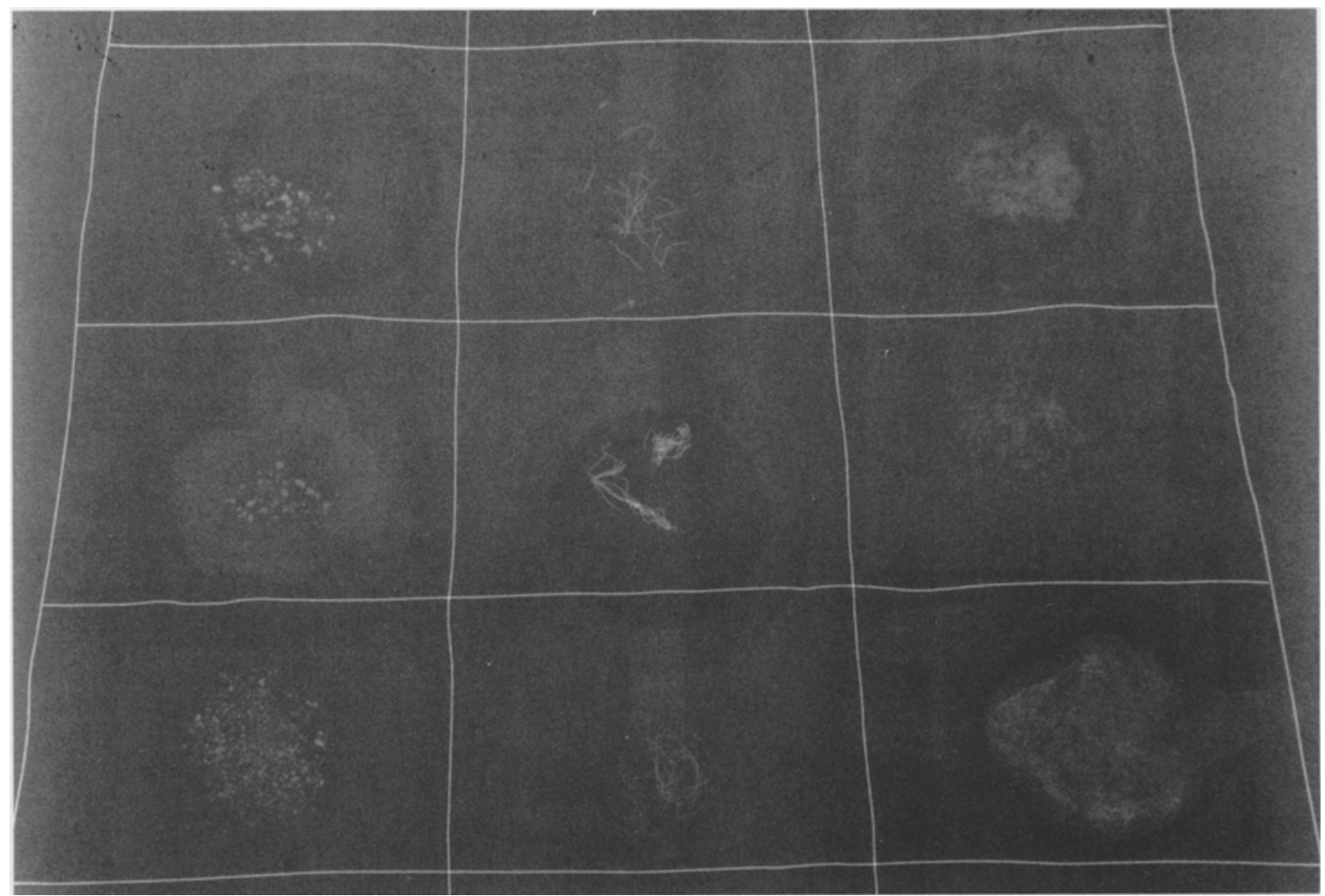

Fig 5. Three type of simulated abnormalities are shown: micronodules (left), linear opacities (center), and groundglass opacities (right).

10-bit depth. So, the pixel size was $0.1 \mathrm{~mm}$. Furthermore, the image size of this FCR system was $14 \times 17$ inches. Observation was performed by eight experienced chest radiologists. For

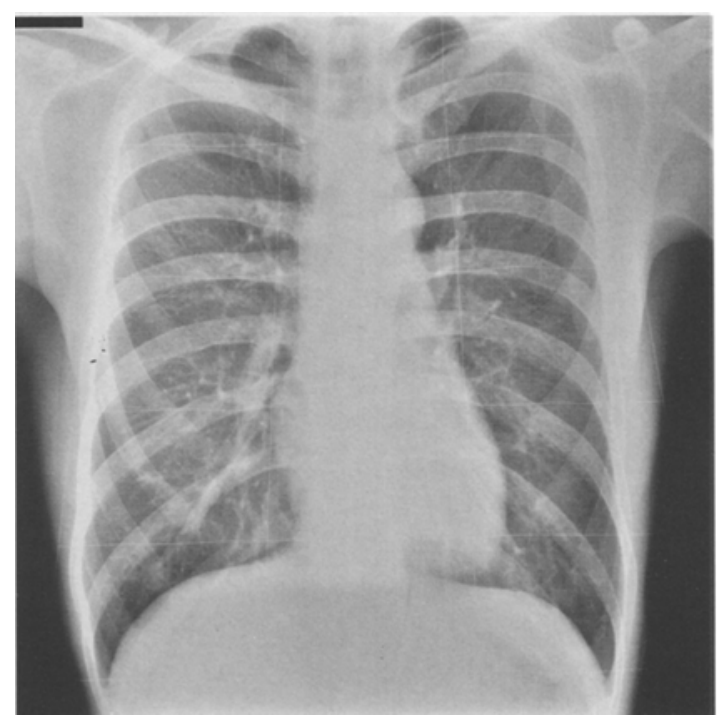

Fig 6. Conventional chest radiograph containing three types of abnormalities. each field divided by copper wire, observers were asked to state the presence or absence of a lesion using a five-level scale of confidence. ROC analysis was performed, and perceptual

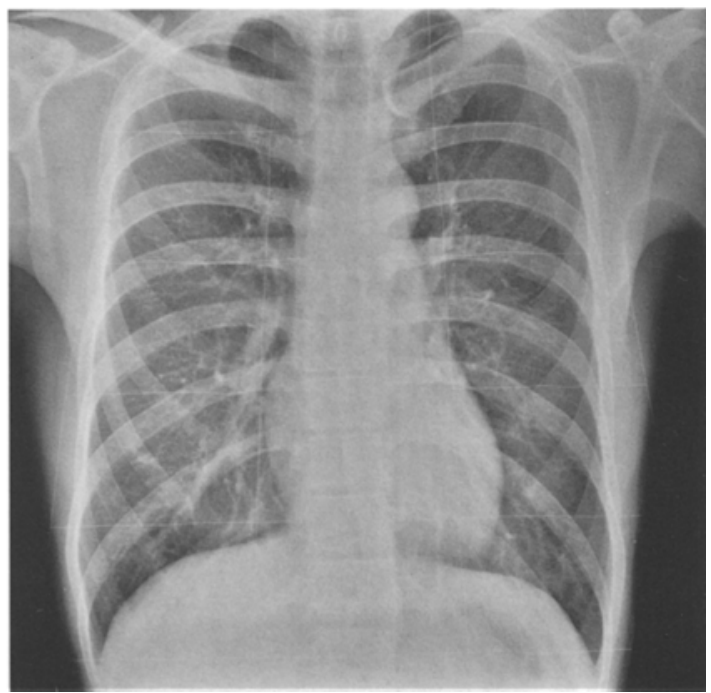

Fig 7. High-resolution storage-phosphor image containing three types of abnormalities. 
accuracy was described by the area under the ROC curve (Az). Az values were used to test the significance of difference in observer performance. All these simulated abnormalities were so subtle that almost all observers indicated score 3 for abnormalities located in the central column and the lowest line. For this reason, the data form the central column and the lowest line were excluded from the final analysis.

There was no significant difference for detecting subtle simulated interstitial abnormalities between conventional screen-film radiography and high-resolution storage-phosphor radiography. For all three types of abnormalities, there was no significant difference between conventional and storage-phosphor radiography (Fig 8).

In conclusion, the high-resolution storagephosphor chest radiography with $0.1-\mathrm{mm}$ pixel size may be substituted for conventional chest radiography in the detection of subtle interstitial abnormalities.

\section{DISCUSSION}

One of the disadvantages of digital chest radiography is its lower spatial resolution compared with conventional screen-film radiography. Because of the status of today's computer technology, extremely large amounts of data

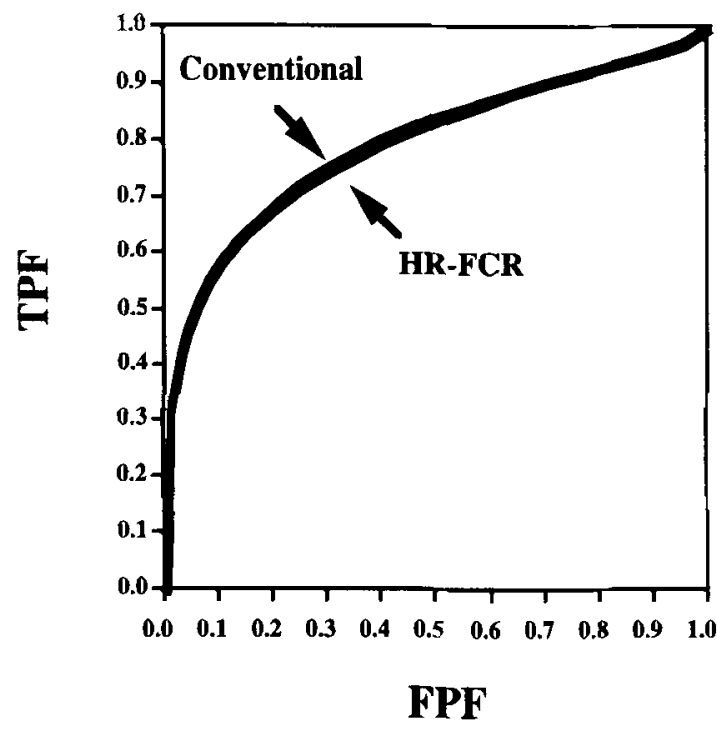

Fig 8. Composite ROC curves of eight chest radiologists. No significant differences in observer performance were observed between conventional film radiographs and storagephosphor images. cannot be managed in a short time. For this reason, data from chest radiography are somewhat difficult to manage without data compression in practical use. This is the main reason why digital chest radiography has lower spatial resolution than conventional screen-film radiography. Although many studies have been published concerning spatial resolution requirements for chest radiography, there is still no world-wide consensus. One approach is to compensate the disadvantage of lower spatial resolution with several types of postprocessing in most of the cases. Furthermore, in addition to postprocessing, this disadvantage can be relieved by changing the concept of a diagnostic work-up for chest disease, eg, much more frequent use of computed tomography (CT), expecially highresolution CT. In patients with some respiratory complaints and with normal chest on digital radiography, CT should be performed. This opinion is supported by most medical physicists, who consider the various advantages of digital imaging rather valuable. Another approach is that disadvantage of lower spatial resolution be considered critical in cases of subtle interstitial lung abnormalities compared with conventional screen-film radiography. Although a postprocessing image is really useful for some types of abnormalities, there is no single postprocessing technique that is efficient for all types of abnormalities. For this reason, several different postprocessing images are necessary to evaluate chest radiography. This is time consuming and not practical. This idea is mainly supported by chest radiologists and chest physicians, especially those in chest disease centers. Thus, they consider that the introduction of a totally digital radiology department remain premature, and that conventional screen film radiography should continue to be used especially for the chest.

From our series of studies, $0.2-\mathrm{mm}$ spatial resolution may be inadequate for diagnosing subtle interstitial lung abnormalities with either storage-phosphor radiography or film-digitized radiography. ${ }^{8,9}$ However, $0.1-\mathrm{mm}$ spatial resolution with life-size display, provided by the FCR 9000 system, may be substituted for conventional radiography. It is very important and impressive that $0.1-\mathrm{mm}$ pixel-size digital chest radiography is commercially available. Furthermore, a life-size image can be obtained. This 
will accelerate the tendency to expand digital imaging in chest radiography. Of course, a high data compression ratio is necessary for image transfer and image storage at present.

\section{REFERENCES}

1. Lams PM, Cocklin ML: Spatial resolution requirement for digital chest radiographs: An ROC study of observer performance in selected cases. Radiology 158:11 19,1986

2. Schaefer CM, Greene RG, Liewellyn HJ, et al: Interstitial lung disease: Impact of postprocessing in digital storage phosphor imaging. Radiology 178:733-738, 1991

3. MacMahon H, Vyborny CJ, Metz CE, et al: Digital radiography of subtle pulmonary abnormalities: An ROC study of the effect of pixel size on observer performance. Radiology 158:21-26, 1986

4. Schaefer CM, Greene R, Oestmann JW, et al: Digital storage phosphor imaging versus conventional film radiography. Radiology 174:207-210, 1990

5. Fajardo LL, Hillman BJ, Pond GD, et al: Detection of pneumothorax: Comparison of digital and conventional chest imaging. AJR 152:475-480, 1989

6. Cox GG, Cook LT, McMillan JH, et al: Chest radiography: Comparison of high-resolution digital displays with conventional and digital film. Radiology 176:771-776, 1990

7. Slasky BS, Gur D, Good WF, et al: Receiver operating characteristic analysis of chest image interpretation with conventional, laser-printed, and high-resolution workstation images. Radiology 174:775-780, 1990

8. Kido S, Ikezoe J, Takeuchi N, et al: Interpretation of subtle interstitial lung abnormalities: Conventional versus storage phosphor radiography. Radiology 187:527-533, 1993

9. Kido S, Ikezoe J, Takeuchi N, et al: Interpretation of subtle interstitial lung abnormalities: Conventional versus film-digitized radiography. Radiology 192:171-176, 1994 